\title{
High prevalence of colonisation with carbapenem-resistant Enterobacteriaceae among patients admitted to Vietnamese hospitals: Risk factors and burden of disease
}

\author{
Dien M. Tran ${ }^{a}$, Mattias Larsson ${ }^{b, c}$, Linus Olson ${ }^{b, c}$, Ngoc T.B. Hoang a, Ngai K. Le ${ }^{a, b}$, \\ Dung T.K. Khu ${ }^{\mathrm{a}, \mathrm{b}}$, Hung D. Nguyen ${ }^{\mathrm{d}}$, Tam V. Vu ${ }^{\mathrm{e}}$, Tinh H. Trinh ${ }^{\mathrm{f}}$, Thinh Q. Le ${ }^{\mathrm{g}}$, \\ Phuong T.T. Phan ${ }^{h}$, Binh G. Nguyen ${ }^{i}$, Nhung H. Pham ${ }^{i}$, Bang H. Mai ${ }^{j}$, Tuan V. Nguyen ${ }^{j}$, \\ Phuong T.K. Nguyen ${ }^{j}$, Nhan D. Le ${ }^{k}$, Tuan M. Huynh ${ }^{1}$, Le T. Anh Thu ${ }^{\mathrm{m}}$, Tran C. Thanh ${ }^{\mathrm{b}}$, \\ Björn Berglund $^{\mathrm{n}}$, Lennart E. Nilsson ${ }^{\mathrm{n}}$, Elin Bornefall ${ }^{\mathrm{n}}$, Le H. Song ${ }^{\mathrm{j}}$, Håkan Hanberger ${ }^{\mathrm{b}, \mathrm{n}, *}$ \\ a Vietnam National Children's Hospital, Hanoi, Vietnam \\ ${ }^{\mathrm{b}}$ Training and Research Academic Collaboration Sweden-Vietnam, Vietnam \\ ${ }^{c}$ Department of Public Health Sciences, Karolinska Institutet, Sweden \\ d St Paul Hospital, Hanoi, Vietnam \\ e Uong Bi Hospital, Quang Ninh, Vietnam \\ ${ }^{\mathrm{f}}$ Binh Dinh General Hospital, Vietnam \\ ${ }^{g}$ Children's Hospital 1, Ho Chi Minh City (HCMC), Vietnam \\ h Phu San Hanoi, Vietnam \\ i Bach Mai Hospital, Hanoi, Vietnam \\ j 108 Military Central Hospital, Hanoi, Vietnam \\ ${ }^{\mathrm{k}}$ Da Nang General Hospital, Vietnam \\ ${ }^{1}$ University Medical Clinic, HCMC, Vietnam \\ ${ }^{\mathrm{m}}$ Cho Ray Hospital, HCMC, Vietnam \\ ${ }^{\mathrm{n}}$ Department of Clinical and Experimental Medicine, Faculty of Medicine and Health Sciences, Linköping University, Sweden
}

\section{A R T I C L E I N F O}

\section{Article history:}

Accepted 16 May 2019

Available online 21 May 2019

\section{Keywords:}

Carbapenem resistance enterobacteriaceae

CRE

Hospital acquired infections

HAI

Colonisation

Mortality
S U M M A R Y

Background: Carbapenem-resistant Enterobacteriaceae (CRE) is an increasing problem worldwide, but particularly problematic in low- and middle-income countries (LMIC) due to limitations of resources for surveillance of CRE and infection prevention and control (IPC).

Methods: A point prevalence survey (PPS) with screening for colonisation with CRE was conducted on 2233 patients admitted to neonatal, paediatric and adult care at 12 Vietnamese hospitals located in northern, central and southern Vietnam during 2017 and 2018. CRE colonisation was determined by culturing of faecal specimens on selective agar for CRE. Risk factors for CRE colonisation were evaluated. A CRE admission and discharge screening sub-study was conducted among one of the most vulnerable patient groups; infants treated at an 80-bed Neonatal ICU from March throughout June 2017 to assess CRE acquisition, hospital-acquired infection (HAI) and treatment outcome.

Results: A total of 1165 (52\%) patients were colonised with CRE, most commonly Klebsiella pneumoniae $(n=805)$, Escherichia coli $(n=682)$ and Enterobacter spp. $(n=61)$. Duration of hospital stay, HAI and treatment with a carbapenem were independent risk factors for CRE colonisation. The PPS showed that the prevalence of CRE colonisation increased on average $4.2 \%$ per day and mean CRE colonisation rates increased from $13 \%$ on the day of admission to $89 \%$ at day 15 of hospital stay. At the NICU, CRE colonisation increased from $32 \%$ at admission to $87 \%$ at discharge, mortality was significantly associated (OR 5.5, $P<0.01$ ) with CRE colonisation and HAI on admission.

\footnotetext{
* Corresponding author at: Division of Infectious Diseases, Department of Clinical and Experimental Medicine, Faculty of Medicine and Health Sciences, Linköping University, 58185 Linköping, Sweden.

E-mail address: hakan.hanberger@liu.se (H. Hanberger).
} 
Conclusion: These data indicate that there is an epidemic spread of CRE in Vietnamese hospitals with rapid transmission to hospitalised patients.

(c) 2019 The Author(s). Published by Elsevier Ltd on behalf of The British Infection Association. This is an open access article under the CC BY-NC-ND license.

(http://creativecommons.org/licenses/by-nc-nd/4.0/)

\section{Introduction}

Antimicrobial resistance is increasing worldwide, eroding the possibility to treat infections ${ }^{1}$. Carbapenem-resistant Enterobacteriaceae (CRE) cause infections that do not respond to standard antibiotic treatment ${ }^{2,3}$. Global spread of CRE is increasing rapidly ${ }^{4-6}$ and $^{7}$ guidelines for infection prevention and control (IPC) measures to prevent this has been prioritised by the $\mathrm{WHO}^{8} \mathrm{ECDC}^{9}$ and CDC. ${ }^{10}$ Except for improvement in IPC measures, development of new antibiotics active against CRE are urgently needed. ${ }^{10,11}$ There is a strong correlation between colonisation with CRE and hospital-acquired infections (HAI) caused by $\mathrm{CRE}^{12-14}$ and it has been shown that CRE colonisation and subsequent infection is associated with increased mortality. ${ }^{15,16}$

High rates of infections caused by extended-spectrum $\beta$ lactamase-(ESBL $\left.\mathrm{A}_{\mathrm{A}+\mathrm{M}}\right)$ producing Enterobacteriaceae, resistant to 3rd generation cephalosporin, but susceptible to carbapenems, have been reported during many years in Southeast Asia. ${ }^{17-19}$ The extensive use of carbapenems has followed suite and the emergence of CRE can thus be anticipated as a result of this. However, data on CRE prevalence in Southeast Asia are scarce. Vietnam has a rapidly developing economy with practically unregulated access to antibiotics through private pharmacies, and the use of antibiotics in the community is widespread, facilitating the emergence of resistant bacteria. Hospitals are crowded and resources for implementation of IPC are often lacking. A HAI prevalence of 30\% was shown in the VINARES survey in intensive care units (ICUs) at 16 Vietnamese hospitals. ${ }^{20,21}$ Klebsiella pneumoniae (of which $55 \%$ were carbapenem-resistant) was the most common aetiology in paediatric ICUs. ${ }^{20}$ In adult ICUs, Acinetobacter baumannii, Pseudomonas aeruginosa and $K$. pneumoniae had carbapenem-resistance rates of $89 \%, 56 \%$, and $15 \%$, respectively. ${ }^{20}$ Whole-genome sequencing of carbapenem-resistant K. pneumoniae (CRKP), revealed a high prevalence of the carbapenemase KPC-2 and increasing colistin resistance due to chromosomal mutations ${ }^{22}$ and the $m c r-1$ gene, ${ }^{23}$ severely limiting treatment options for serious HAIs caused by $K$. pneumoniae. The high frequency of $\mathrm{CRKP}^{20}$ initiated further surveillance of CRE colonisation to determine the dissemination at country, region, hospital and ward levels.

The aims of this study were to study the prevalence and risk factors for CRE colonisation among patients admitted to Vietnamese hospitals, and to measure the transmission and burden of CRE among new-born children admitted to a neonatal ICU.

\section{Methods}

Carbapenem-resistant enterobacteriaceae point prevalence study (CRE-PPS)

\section{Setting}

Surveys were conducted in 63 wards at 12 Vietnamese hospitals. The participating hospitals were largely recruited from the previous VINARES study network, with a geographic spread covering northern, central and southern Vietnam. ${ }^{20,21}$

\section{Study performance}

To assess the epidemiology of CRE, screening of CRE carriage was performed among patients admitted to participating units by rectal swabs. The surveys were conducted from March 2017 through September 2018. In each participating hospital the PPS was conducted during 1-3 days depending on the capacity of the clinical microbiological laboratory.

The surveys were made in collaboration with each hospital and its infection control unit. A basic standard case report form (CRF), designed according to the needs of the hospital, was used. The following information was requested from all patients: type of ward and number of days of admission on the day of screening. An extended protocol was used for a subset of patients including the following data: patient ID, gender, date of birth, source of admission, reason for admission, infection diagnosis, HAI, invasive procedures, and antibiotic treatment. These latter data were used for analysis of risk factors for CRE colonisation. The research team provided training for screening and clinical data collection as well as guidance during implementation. Allocated hospital staff conducted the screening and laboratory work.

Inclusion criteria: Patients treated at/admitted to a participating hospital ward on the day of the CRE-PPS.

Exclusion criteria: Anal atresia or other reasons for failure to collect the rectal swab sample on the day of screening or non-consent by adult patient, caretaker, legal guardian or relative. In the ICU setting, consent was on opt-out basis as it was considered important from the infection control point of view to have as complete data as possible regarding CRE colonisation. All patients and/or relatives were informed about the screening process and their right to decline participation.

\section{Sub-study: transmission and burden of CRE on an 80-bed NICU}

Setting: An 80-bed neonatal ICU (NICU) in a tertiary care paediatric hospital in northern Vietnam serving a population of approximately 40 million people.

Performance of the study: Faecal specimens were collected by rectal swabs on admission and discharge of all admitted neonates between March and June 2017. The samples were analysed at the hospital's clinical microbiological laboratory. The following data were collected from each patient: patient ID, gender, date of birth, source of admission, reason for admission, infection diagnosis, HAI, invasive procedures, antibiotic treatment and treatment outcome. Rates of HAI and treatment outcome in relation to CRE colonisation were determined.

Inclusion criteria: The inclusion criterion was admission to the NICU during the study period.

Exclusion criteria: Anal atresia or other reason for failure to collect the rectal swab sample on the day of screening, opt out consent by caretaker, legal guardian or relative.

Mortality: Mortality was defined as death in hospital or withdrawal from treatment due to pessimal prognosis with no further treatment options.

\section{Definitions}

HAI was defined according to the European Centre for Disease Control and Prevention (ECDC), as used in the previous VINARES study. ${ }^{20,24}$ 


\section{Microbiological analysis}

Faecal samples were collected on ESwabs (Copan, Brescia, Italy), transported to the laboratory, and plated on Chrom ID Carba agar plates (BioMérieux, Marcy-l'Étoile, France). ${ }^{25,26}$ The samples were incubated for 16-24 h before reading. In the CRE-PPS, species determination was performed with VITEK 2 (BioMérieux) and susceptibility testing was performed by using either VITEK 2 or disk diffusion, depending on the routine in the respective laboratory and in agreement with CLSI guidelines. In the sub-study on the 80-bed NICU, only VITEK 2 was used for species determination and susceptibility testing according to CLSI guidelines. The microbiological analyses were supervised by the study team in all laboratories. A photo was taken from all plates, stored and submitted to the study team in case of uncertainty.

Statistical analysis

CRE-PPS: Risk factors for CRE colonisation were analysed with univariate analysis using $\chi^{2}$-tests, odds ratio (OR), and 95\% confidence intervals $(\mathrm{CI})$. All significant variables in the univariate analysis $(P<0.05)$ were included in a multivariate regression analysis using binary logistic regression. The analysis was based on cases of unique patients. A p-value $<0.05$ was considered significant. SPSS 22.0 was used for all statistical calculations.

Sub-study: Transmission and burden of CRE on an 80-bed NICU: Since CRE on admission and HAI at discharge were correlated, we stratified by CRE on admission and constructed two separate logistic regression models. Analysis was performed to assess risk factors for mortality in relation to CRE colonisation using uni- and multivariate analyses.

\section{Ethical approval}

Ethical approval was obtained from the Vietnam National Children Hospital (VNCH) Ethics Committee reference number 812/QDBVNTU, 23rd March 2017. The VNCH Ethics Committee is a subcommittee of the Ministry of Health Ethics Council and its approval extends to other hospitals in Vietnam. The ethics committees of all hospitals participating in the survey were provided with and accepted the VNCH's ethical approval.

\section{Results}

CRE-PPS

Of the 2233 patients screened, 1165 (52\%) were colonised with CRE (Table 1). The most frequent bacterial species were K. pneumoniae followed by E. coli and Enterobacter spp (Table 2).

The CRE colonisation prevalence was $13 \%$ among patients admitted the same day as the screening was performed (Day 0). Corresponding prevalence figures were $45 \%$ on Day 3 and $89 \%$ on Day 15. The prevalence of CRE colonisation increased on average $4.2 \%$ per day (Fig. 1).

CRE colonisation rates differed between levels of care and was highest on the ICUs (Tables 1 and 2) followed by general wards, adult and paediatric emergency wards (Tables 1 and 2). Sixty-four percent of the patients admitted to an ICU were colonised with CRE (Table 1). The highest CRE colonisation rate (83\%) was reported from a central hospital NICU and the lowest (4\%) at an adult emergency care unit where most patients entered the ward directly from the community (Table 2). At one central hospital NICU, screening for colonisation with CRE was performed twice, the first in 2017 and the second in 2018. The frequency of colonisation on this NICU increased from $67 \%$ to $83 \%$ over the 12 -month period. A
Table 1

CRE colonisation according to level of care and type of department

\begin{tabular}{llllll}
\hline Patient type & Variable & ICU & Ward & Emergency & Total \\
\hline Neonatal & N Total & 247 & & & 247 \\
& N CRE+ & 161 & & & 161 \\
& \%CRE+ & $\mathbf{6 5}$ & & & $\mathbf{6 5}$ \\
& N KP & 99 & & & 99 \\
& N EC & 89 & & & 89 \\
Paediatric & N Total & 391 & 772 & 56 & 1219 \\
& N CRE+ & 270 & 376 & 9 & 655 \\
& \%CRE+ & $\mathbf{6 9}$ & $\mathbf{4 9}$ & $\mathbf{1 6}$ & $\mathbf{5 4}$ \\
& N KP & 171 & 342 & 4 & 517 \\
Adult & N EC & 117 & 347 & 7 & 471 \\
& N Total & 392 & 318 & 57 & 767 \\
& N CRE+ & 232 & 115 & 2 & 349 \\
& \%CRE+ & $\mathbf{5 9}$ & $\mathbf{3 6}$ & $\mathbf{4}$ & $\mathbf{4 6}$ \\
& N KP & 120 & 67 & 2 & 189 \\
Total & N EC & 72 & 50 & 0 & 122 \\
& N Total & 1030 & 1090 & 113 & 2233 \\
& N CRE+ & 663 & 491 & 11 & 1165 \\
& \%CRE+ & $\mathbf{6 4}$ & $\mathbf{4 5}$ & $\mathbf{1 0}$ & 52 \\
& N KP & 390 & 409 & 6 & 805 \\
& N EC & 278 & 397 & 7 & 682 \\
& & &
\end{tabular}

CRE colonisation among 2233 patients was evaluated by data collected from PPS performed at 12 Vietnamese hospitals. The data are sorted according to CRE colonisation prevalence in relation to level of care and type of department.

$N=$ number, $\mathrm{KP}=K$. pneumoniae, $\mathrm{EC}=E$. coli.

similarly rapid increase in colonisation was observed on a paediatric provincial hospital, increasing from $29 \%$ to $53 \%$ in 16 months, and on an adult ICU where the frequency of CRE colonisation increased from $54 \%$ to $71 \%$ in 8 months (Table 2).

Characteristics of the patients and univariate analysis of risk factors for CRE colonisation are shown in Table 3A. Multivariate analyses of risk factors for CRE colonisation are based on complete care data from 1173 of all 2233 patients showed the following significant risk factors for colonisation with CRE: long duration of hospital stay, HAI and treatment with a carbapenem (Table 3B).

\section{Sub-study: transmission and burden of CRE on an 80-bed NICU}

Of a total of 328 neonates, $279(85 \%)$ were referred from another hospital, where the average length of stay in the referred hospital before admission to the NICU was 8.2 days. The prevalence of colonisation with CRE increased from 32\% at ICU admission to $88 \%$ at discharge. On average, neonates colonised with CRE had 9 days longer hospital stay. Patient characteristics are shown in Table 4 . The length of NICU stay was significantly $(P<0.001)$ longer for patients colonised with CRE on admission and discharge (16.4 and 14.9 days, respectively), compared to non-CRE colonised patients on admission and discharge (9.3 and 3.2 days, respectively). The overall mortality was $25 \%$, HAI on admission and HAI at discharge were significantly and independently associated with mortality $(P<0.01)$. Mortality was significantly higher (univariate analysis) among patients CRE colonised at admission compared to non-colonised, $43 \%$ and $28 \%$, respectively (Table 4 ). CRE colonisation is a prerequisite for infection caused by CRE. It cannot be expected, to be a causal relationship between CRE-colonisation itself and mortality. Thus, since CRE at admission and HAI at discharge were correlated, we stratified by CRE on admission and performed two separate logistic regression models. When stratified by CRE colonisation on admission, there was more than a five-fold increased mortality risk for patients with CRE colonisation and HAI on admission as compared to no independent increased risk for patients negative for CRE but HAI on admis- 
Table 2

CRE colonisation among 2233 patients was evaluated by data collected from PPSs performed at 12 Vietnamese hospitals.

\begin{tabular}{|c|c|c|c|c|c|c|c|c|c|c|c|}
\hline Hospital & Type & Location & Level & Area & Year & $\mathrm{CRE}+(\mathrm{N})$ & All $(\mathrm{N})$ & CRE + (\%) & $\mathrm{KP}(\mathrm{N})$ & EC $(N)$ & EB $(N)$ \\
\hline 1 & $\mathrm{NeO}$ & Central & $\mathrm{NICU}(1)^{*}$ & North & 2018 & 49 & 59 & 83 & 39 & 28 & 5 \\
\hline 2 & Neo & City & NICU (1) & North & 2017 & 32 & 41 & 78 & 16 & 17 & 4 \\
\hline 1 & Ped & Central & ICU's (3) & North & 2018 & 37 & 50 & 74 & 19 & 13 & 8 \\
\hline 6 & Ped & Central & PICU (2) & South & 2017 & 33 & 45 & 73 & 27 & 14 & \\
\hline 1 & Ped & Central & ICU's (6) & North & 2017 & 178 & 246 & 72 & 110 & 84 & 6 \\
\hline 11 & Adult & City & ICU (1)\# & South & 2018 & 30 & 42 & 71 & 25 & 16 & \\
\hline 5 & Adult & Provincial & ICU (1) & Middle & 2018 & 29 & 42 & 69 & 12 & 15 & 16 \\
\hline 12 & Adult & Central & ICU (1) & South & 2017 & 22 & 32 & 69 & & & \\
\hline 2 & Adult & City & MICU (1) & North & 2017 & 25 & 37 & 68 & 14 & 6 & \\
\hline 1 & Neo & Central & $\operatorname{NICU}(1)^{*}$ & North & 2017 & 39 & 58 & 67 & 38 & 11 & 1 \\
\hline 10 & Adult & Provincial & ICU (1) & Middle & 2017 & 37 & 60 & 62 & 23 & 6 & \\
\hline 8 & Adult & Central & MICU (1) & North & 2017 & 24 & 40 & 60 & 21 & 5 & \\
\hline 1 & Ped & Central & Wards (28) & North & 2017 & 230 & 395 & 58 & 244 & 274 & 9 \\
\hline 5 & Ped & Provincial & PICU (1) & Middle & 2018 & 11 & 19 & 58 & 5 & 4 & 2 \\
\hline 2 & Adult & City & SICU (1) & North & 2017 & 18 & 33 & 55 & 8 & 6 & \\
\hline 11 & Adult & City & ICU (1)\# & South & 2018 & 22 & 41 & 54 & 10 & 7 & \\
\hline 3 & Ped & Provincial & Wards $(10)^{* *}$ & North & 2018 & 95 & 178 & 53 & 69 & 49 & \\
\hline 9 & Adult & Central & Wards (4) & North & 2017 & 21 & 43 & 49 & 17 & 6 & 1 \\
\hline 7 & Neo & City & NICU (1) & North & 2017 & 34 & 73 & 47 & 1 & 33 & \\
\hline 5 & Neo & Provincial & NICU (1) & Middle & 2018 & 7 & 16 & 44 & 5 & & 1 \\
\hline 9 & Adult & Central & MICU (1) & North & 2017 & 25 & 65 & 38 & 7 & 11 & \\
\hline 11 & Adult & City & Wards (14) & South & 2018 & 90 & 240 & 38 & 46 & 44 & \\
\hline 2 & Ped & City & PICU (1) & North & 2017 & 11 & 31 & 35 & 10 & 2 & 1 \\
\hline 3 & Ped & Provincial & Wards $(10)^{* *}$ & North & 2017 & 34 & 118 & 29 & 21 & 17 & \\
\hline 4 & Ped & Provincial & Wards (2) & North & 2017 & 17 & 81 & 21 & 8 & 7 & 7 \\
\hline 1 & Ped & Central & Emergency (1) & North & 2017 & 9 & 56 & 16 & 4 & 7 & \\
\hline 9 & Adult & Central & Wards (3) & North & 2017 & 4 & 35 & 11 & 4 & & \\
\hline 9 & Adult & Central & Emergency (1) & North & 2017 & 2 & 57 & 4 & 2 & & \\
\hline Total & & & & & & 1165 & 2233 & 52 & 805 & 682 & 61 \\
\hline
\end{tabular}

The data are sorted according to CRE colonisation in relation to hospital, patient group, hospital location, level of care, area and year of study. $N=$ number $\mathrm{KP}=K$. pneumoniae $\mathrm{EC}=E$. coli $\mathrm{EB}=$ Enterobacter spp. Repeated PPS: ${ }^{*} \mathrm{NICU} 12$ months apart; **Provincial paediatric hospital 16 months apart; \#Adult ICU 8 months apart.

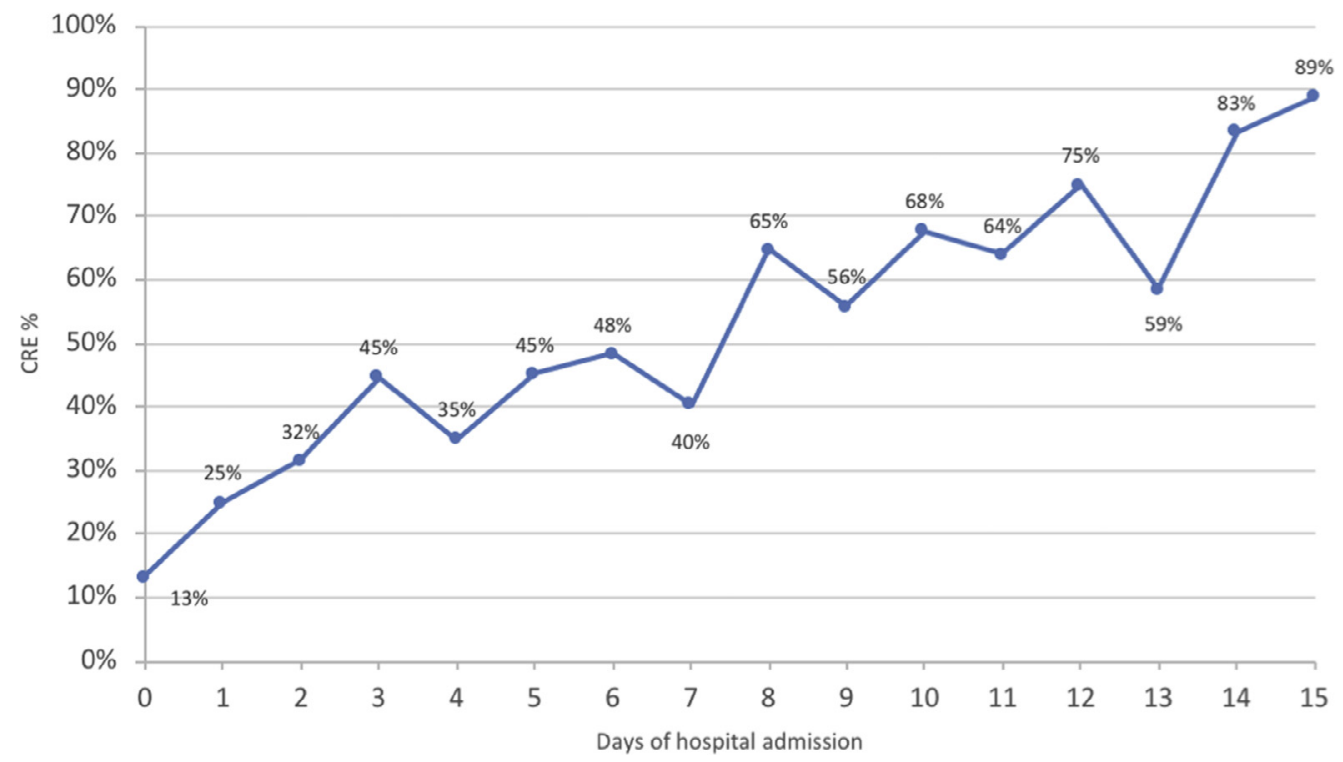

Fig. 1. CRE prevalence in relation to days of hospital admission.

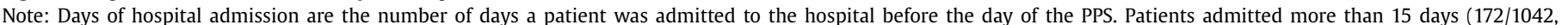
$16.5 \%$ ) were not included in the analysis.

sion (Table 5). There was a 3.7-fold independent increased mortality risk for patients negative for CRE on admission but suffering from HAI and colonised with CRE at discharge (Table 5). All patients diagnosed with HAI were colonised with CRE at discharge. The most common antibiotics used prior to admission were cephalosporins followed by aminoglycosides and carbapenems. The most common antibiotics used during hospital stay were aminoglycosides followed by cephalosporins, penicillins, carbapenems and colistin.

\section{Discussion}

\section{Main findings}

This study showed that a mean of $52 \%$ of patients admitted to hospitals throughout Vietnam were colonised with CRE. Mean CRE colonisation rates increased from $13 \%$ on the day of admission to $89 \%$ at Day 15 of hospital stay. Apart from long duration of hospital stay, risk factors for CRE colonisation were HAI, and 
Table 3A

Risk factors for CRE colonisation $(n=1.173)$ included in PPSs performed at 12 Vietnamese hospitals, determined by univariate statistics.

\begin{tabular}{|c|c|c|c|c|c|c|}
\hline Variable & CRE- & & CRE + & & All & $p$-value \\
\hline Average age (years) & 33.9 & & 38.9 & & 34.4 & $<0.01$ \\
\hline Duration hospital stay (days) & 5.5 & & 16.2 & & 9.9 & $<0.01$ \\
\hline Variable & $\mathrm{CRE}-(\mathrm{N})$ & CRE- (\%) & $\mathrm{CRE}+(\mathrm{N})$ & $\mathrm{CRE}+(\%)$ & Total (N) & $p$-value \\
\hline Male & 296 & 52 & 274 & 48 & 570 & 0.65 \\
\hline Female & 189 & 53 & 165 & 47 & 354 & 0.65 \\
\hline Admission Hospital & 211 & 50 & 213 & 50 & 424 & 0.32 \\
\hline$<48 \mathrm{~h}$ in hospital & 275 & 61 & 175 & 39 & 450 & $<0.01$ \\
\hline$>48 \mathrm{~h}$ in hospital & 247 & 47 & 280 & 53 & 527 & $<0.01$ \\
\hline K. pneumoniae & 0 & 0 & 260 & 100 & 260 & $<0.01$ \\
\hline E. coli & 0 & 0 & 179 & 68 & 263 & $<0.01$ \\
\hline Children & 295 & 58 & 213 & 42 & 508 & .03 \\
\hline Adults & 310 & 47 & 355 & 53 & 665 & .03 \\
\hline Pre-term & 40 & 33 & 80 & 67 & 120 & $<0.01$ \\
\hline Full-term & 69 & 67 & 34 & 33 & 103 & $<0.01$ \\
\hline Caesarean & 42 & 65 & 23 & 35 & 65 & .82 \\
\hline Vaginal & 65 & 66 & 33 & 34 & 98 & .82 \\
\hline ICU & 304 & 46 & 350 & 54 & 654 & $<0.01$ \\
\hline Wards & 300 & 58 & 218 & 42 & 518 & $<0.01$ \\
\hline Neonatal ICU & 98 & 47 & 111 & 53 & 209 & 0.14 \\
\hline Paediatric ICU & 62 & 47 & 69 & 53 & 131 & 0.30 \\
\hline Paediatric wards & 134 & 80 & 33 & 20 & 167 & $<0.01$ \\
\hline ICU-Adult & 154 & 47 & 173 & 53 & 327 & 0.06 \\
\hline Adult wards & 447 & 54 & 387 & 46 & 834 & 0.03 \\
\hline $\mathrm{HAI}$ & 66 & 31 & 144 & 69 & 210 & $<0.01$ \\
\hline Peumonia HAI & 31 & 32 & 65 & 68 & 96 & $<0.01$ \\
\hline Sepsis HAI & 14 & 27 & 38 & 73 & 52 & $<0.01$ \\
\hline CVC & 84 & 48 & 92 & 52 & 176 & 0.65 \\
\hline PVC & 345 & 50 & 351 & 50 & 696 & 0.22 \\
\hline Intubation & 132 & 40 & 201 & 60 & 333 & $<0.01$ \\
\hline Carbapenem use & 56 & 30 & 130 & 70 & 186 & $<0.01$ \\
\hline Cephalosporin use & 260 & 53 & 229 & 47 & 489 & 0.13 \\
\hline Aminoglycoside use & 93 & 48 & 100 & 52 & 193 & 0.48 \\
\hline Fluoroquinolone use & 62 & 39 & 98 & 61 & 160 & $<0.01$ \\
\hline Colistin use & 9 & 22 & 32 & 78 & 41 & $<0.01$ \\
\hline
\end{tabular}

Table 3B

Multivariate analysis.

\begin{tabular}{|c|c|c|c|c|c|c|c|c|c|}
\hline Variable & CRE- & & CRE+ & & All & Multivariate $p$-value** & Odds ratio & CI $95 \%$ & CI 95\% \\
\hline Treatment-time (days) & 5.5 & & 16.2 & & 9,9 & $<0.01$ & 1.033 & 1.019 & 1.047 \\
\hline Variable & $\mathrm{CRE}-(\mathrm{N})$ & CRE- $(\%)$ & $\mathrm{CRE}+(\mathrm{N})$ & $\mathrm{CRE}+(\%)$ & Total $(\mathrm{N})$ & Multivariate $p$-value $e^{* *}$ & Odds ratio & CI 95\% & CI $95 \%$ \\
\hline Children & 295 & 58 & 213 & 42 & 508 & .187 & .803 & .580 & 1.112 \\
\hline ICU & 304 & 46 & 350 & 54 & 654 & .53 & .90 & .65 & 1.26 \\
\hline HAI & 66 & 31 & 144 & 69 & 210 & .01 & 1.74 & 1.16 & 2.59 \\
\hline Intubation & 132 & 40 & 201 & 60 & 333 & .17 & 1.25 & .91 & 1.72 \\
\hline Carbapenem use & 56 & 30 & 130 & 70 & 186 &.$<0.01$ & 1.79 & 1.20 & 2.68 \\
\hline Fluoroquinolon use & 62 & 39 & 98 & 61 & 160 & .91 & .98 & .65 & 1.46 \\
\hline Colistin use & 9 & 22 & 32 & 78 & 41 & .52 & 1.31 & .57 & 3.02 \\
\hline
\end{tabular}

Risk factors for CRE colonisation $(n=1.173)$ included in PPSs performed at 12 Vietnamese hospitals, determined by multivariate analysis. To sustain the power for multivariate analysis larger compound variables were created including ICU vs wards as well as adults vs children.

carbapenem treatment. This virtually nationwide screening survey at 12 hospitals in Vietnam including 2233 patients indicated that the rapid colonisation with CRE among Vietnamese in-patients has reached epidemic proportions. Mortality risk associated with CRE colonisation was measured among one of the most vulnerable patient groups; infants treated on a NICU in a tertiary care hospital. Neonates that already had HAI and CRE colonisation on transfer to the NICU had an increased mortality risk which agrees with other studies. ${ }^{15,27-29}$ CRE colonisation places a major burden on the healthcare system due to the increased risk of HAI caused by CRE and associated increased mortality. ${ }^{12-15,29}$

As yet there are no coordinated programmes for hospital admission screening for multidrug resistant bacteria, including CRE, in Vietnam, Hence, data on resistance are based on reports of clinical cultures that may be biased due to skewed selection of cul- tures (e.g. overrepresentation of severe patients and those with treatment failure), but this PPS reflects the true prevalence of CRE colonisation in Vietnamese hospitals.

The current situation at Vietnamese hospitals with about half of hospital patients being colonised with CRE indicates a widespread CRE problem. Similar high colonisation rates have previously been reported from single centres, and a few countries but data are scarce from South East Asia. ${ }^{15,30,31}$ ). Increased hospital stay and mortality was shown to be associated with CRE infection in a recent published study. ${ }^{32}$ In hospitals that were screened both 2017 and 2018, the increase in CRE prevalence was considerable. This indicates that improvement in IPC measures is urgent, otherwise we may not have seen the peak of this hospital epidemic. We did not consider the prevalence of CRE in the community in this study, but data from the emergency ward shortly after admission showed 
Table 4

Admission and discharge screening for CRE colonisation was performed on 329 patients on an 80-bed NICU at a Vietnamese hospital.

\begin{tabular}{|c|c|c|c|c|c|c|}
\hline & Survived (N) & Survived (\%) & Mortality (N) & Mortality (\%) & Total $(\mathrm{N})$ & Univariate $p$-value* \\
\hline $\mathrm{CRE}+$ admission & 46 & 57 & 35 & 43 & 81 & 0.01 \\
\hline $\mathrm{CRE}+$ discharge & 154 & 73 & 58 & 27 & 212 & $0 \cdot 18$ \\
\hline CRE- discharge & 26 & 84 & 5 & 16 & 31 & \\
\hline CRE acquisition & 109 & 78 & 30 & 22 & 139 & $0 \cdot 17$ \\
\hline Male & 144 & 72 & 55 & 28 & 199 & \\
\hline$\geq 7$ days treatment & 56 & 62 & 34 & 38 & 90 & $<0.01$ \\
\hline$<7$ days treatment & 165 & 81 & 38 & 19 & 203 & \\
\hline Preterm & 87 & 65 & 46 & 35 & 133 & $<0.01$ \\
\hline Full-term & 146 & 82 & 32 & 18 & 178 & \\
\hline Caesarean section & 115 & 80 & 28 & 20 & 143 & $0 \cdot 11$ \\
\hline Pneumonia & 51 & 76 & 16 & 24 & 67 & 0.77 \\
\hline Sepsis & 30 & 65 & 16 & 35 & 46 & $0 \cdot 11$ \\
\hline PDA & 8 & 57 & 6 & 43 & 14 & 0.12 \\
\hline Asphyxia & 5 & 83 & 1 & 17 & 6 & 0.63 \\
\hline Intraventricular haemorrhage & 4 & 100 & 0 & 0 & 4 & 0.24 \\
\hline Gastroenteritis & 9 & 100 & 0 & 0 & 9 & 0.08 \\
\hline $\mathrm{AB}$ use before admission & 146 & 73 & 53 & 27 & 199 & 0.46 \\
\hline HAI admission & 46 & 53 & 40 & 47 & 86 & $<0.01$ \\
\hline KPC & 32 & 58 & 23 & 42 & 55 & 0.01 \\
\hline $\mathrm{KP}$ resistant to Gentamicin & 25 & 60 & 17 & 40 & 42 & 0.08 \\
\hline KP resistant to Amikacin & 19 & 54 & 16 & 46 & 35 & 0.01 \\
\hline Carbapenems & 51 & 61 & 32 & 39 & 83 & $<0.01$ \\
\hline Aminoglycosides & 118 & 78 & 33 & 22 & 151 & 0.12 \\
\hline Colistin & 6 & 29 & 15 & 71 & 21 & $<0.01$ \\
\hline Culture confirmed HAI & 25 & 56 & 20 & 44 & 45 & $<0.01$ \\
\hline HAI discharge & 64 & 55 & 52 & 45 & 116 & $<0.01$ \\
\hline HAI pneumonia & 48 & 58 & 35 & 42 & 83 & $<0.01$ \\
\hline HAI sepsis & 34 & 55 & 28 & 45 & 62 & $<0.01$ \\
\hline
\end{tabular}

Patient characteristics and CRE colonisation are shown in relation to mortality.

RDS - Respiratory Distress Syndrome, PDA - Persistant Ductus Arteriousus, KP $=$ K. pneumoniae, CVC-Central Venous Catheter, PVCPeripheral Venous Catheter, $\mathrm{AB}$ - Antibiotic.

Table 5

CRE colonisation and mortality among patients admitted to an 80-bed NICU during March-May 2017.

\begin{tabular}{|c|c|c|c|c|c|c|c|c|}
\hline \multirow[t]{2}{*}{ CRE admission } & & \multirow[t]{2}{*}{ Crude mortality (N) } & \multirow[t]{2}{*}{ Survived (N) } & \multirow[t]{2}{*}{ Crude mortality (\%) } & \multirow[t]{2}{*}{ Sig. } & \multirow[t]{2}{*}{ OR } & \multicolumn{2}{|c|}{ 95\% C.I. } \\
\hline & & & & & & & Lower & Upper \\
\hline \multirow[t]{8}{*}{ Negative } & HAI admission & 16 & 30 & 35 & .98 & 0.99 & .40 & 2.45 \\
\hline & No HAI & 32 & 142 & 18 & & & & \\
\hline & HAI discharge & 25 & 21 & 54 & $<0.01$ & 3.71 & 1.61 & 8.56 \\
\hline & No HAI & 40 & 133 & 23 & & & & \\
\hline & Preterm & 27 & 59 & 31 & .03 & 2.57 & $1 \cdot 1+$ & 6.02 \\
\hline & Full term & 18 & 108 & 14 & & & & \\
\hline & Admission $\geq 7$ days & 17 & 40 & 30 & .96 & 1.03 & .43 & 2.43 \\
\hline & Admission $<7$ days & 24 & 118 & 17 & & & & \\
\hline \multirow[t]{8}{*}{ Positive } & HAI admission & 24 & 11 & 69 & $<0.01$ & 5.48 & 1.84 & $16 \cdot 35$ \\
\hline & No HAI & 14 & 52 & 21 & & & & \\
\hline & HAI discharge & 23 & 43 & 50 & .02 & 4.05 & 1.28 & 12.89 \\
\hline & No HAI & 8 & 27 & 23 & & & & \\
\hline & Preterm & 19 & 28 & 40 & .51 & 1.41 & .52 & 3.85 \\
\hline & Full-term & 14 & 38 & 27 & & & & \\
\hline & Admission $\geq 7$ days & 17 & 16 & 52 & .34 & 1.69 & .57 & 4.99 \\
\hline & Admission $<7$ days & 14 & 47 & 23 & & & & \\
\hline
\end{tabular}

Multivariate analysis: Since the effect of HAI at discharge is modified by CRE on admission, we stratified by CRE on admission and performed two separate logistic regression models (see the table). 
a significantly lower prevalence compared to that on the general ward and ICU. The high CRE colonisation rate among hospital patients results in a high risk for spread within the family and community when CRE colonised patients are discharged. ${ }^{33}$ Implementation of IPC strategies is particularly important when neonates are discharged, since parents and others in the household take care of them and their faeces (changing diapers), which may lead to transmission of CRE within the family and near environment.

What can be done to reduce colonisation and prevent infections caused by CRE? - WHO, ECDC ${ }^{9}$ and CDC have published guidelines and recommend multimodal IPC strategies for carbapenem-resistant Gram-negative bacteria. ${ }^{8-10,34}$ Early detection of CRE colonisation is crucial, as is isolation in a single room or cohort care with patients colonised by the same strain. This multimodal strategy has been successful in one high income country (Israel), reducing the spread of CRE in both acute care hospitals and long-term care facilities ${ }^{35,36}$. In Vietnam, a lower-middleincome country with limited resources, cohort care is the only option due to lack of isolation rooms and a very high prevalence of CRE-colonised patients.

CRE infections are difficult to treat and the risk of treatment failure is high. ${ }^{27,28}$ Thus, rapid cost-effective microbiological diagnostics to reduce therapeutic failure and optimisation of treatment for patients with a severe Gram-negative bacterial infection is needed. Unfortunately, this is not available in most hospitals in Vietnam, but by faecal screening, CRE-colonised patients which have increased risk of nosocomial infections due to CRE can get earlier appropriate treatment against HAI.

The strength of this PPS study was the large number of patients included, providing significant results regarding prevalence of and risk factors for CRE colonisation. We performed the study using selective ESBL-carba plates at a very low cost. There are several limitations of the study including the lack of genotyping data from all hospitals, but this was not feasible in the PPS-study due to cost and limited laboratory capacity. Some of the hospitals could not provide all clinical data in time for the manuscript preparation and was not included in the risk analysis for CRE-colonisation, but these analyses are based on 1173 patients with clinical data, thus not biased by delayed reporting. Another limitation is that we included mainly national level hospitals and departments with high risk for nosocomial infections e.g. ICU's and these data are not representative for all levels of care in Vietnam. However, the PPS data are representative for the wards assessed and possibly for other similar wards at the same level of care. We were not able to provide comprehensive data on the aetiology of HAI due to low compliance in taking cultures before starting antibiotic treatment. However, previous studies have shown that there is a strong correlation between colonisation with CRE and HAI caused by CRE. ${ }^{12-14}$

In this paper we report an epidemic spread of CRE on 63 wards in 12 Vietnamese hospitals. Colonisation of hospitalised patients is rapid, increasing for each day of hospitalisation. CRE colonisation and HAI are associated with increased mortality, increased risk of treatment failure and thereby healthcare costs. IPC measures including cohort care should be implemented to limit HAIs caused by CRE.

This study shows that large-scale epidemiological surveillance of CRE using affordable methods is possible in low- and middleincome countries. It is imperative that awareness of the epidemiological situation is raised if we are to see an increase in the implementation of IPC strategies.

\section{Conflicts of interest}

There is no reported conflict of interest. All representatives from the anonymous hospitals have agreed to participate in the paper.

\section{Acknowledgment}

We thank all Vietnamese hospitals and colleagues for participating in the CRE screening study. We would like to give warm thanks to: Mats Fredriksson, senior lecturer, statistician at Linköping University for advice and statistical analysis; Rogier van Doorn, director at Oxford Clinical Research Unit in Hanoi for valuable comments on the report, Maud Nilsson, Linköping University being helpful with laboratory materials sent to Vietnam; Annelie Brauner, professor emerita Karolinska for introducing master students in data collection.

These studies were funded by the Karolinska Institute, Solna, Sweden, Linköping University, County of Östergötland, Sweden, Swedish Foundation for International Cooperation in Research and Higher Education, STINT, Stockholm, Sweden, European Union, Marie Slodowska Curie Grants, and ReAct, Uppsala, Sweden, as well as the participating hospitals.

\section{References}

1. Cassini A, Hogberg LD, Plachouras D, Quattrocchi A, Hoxha A, Simonsen GS, et al. Attributable deaths and disability-adjusted life-years caused by infections with antibiotic-resistant bacteria in the EU and the european economic area in 2015: a population-level modelling analysis. Lancet Infect Diseas 2019:19(1):56-66.

2. Martin A, Fahrbach K, Zhao Q, Lodise T. Association between carbapenem resistance and mortality among adult, hospitalized patients with serious infections due to enterobacteriaceae: results of a systematic literature review and metaanalysis. Open Forum Infect Dis 2018;5(7) ofy150.

3. Nabarro LEB, Shankar C, Pragasam AK, Mathew G, Jeyaseelan V, Veeraraghavan B, et al. Clinical and bacterial risk factors for mortality in children with carbapenem-resistant enterobacteriaceae bloodstream infections in India. Pediatr Infect Diseas J 2017;36(6):e161-e1e6.

4. Brolund A, Lagerqvist N, Byfors S, Struelens MJ, Monnet DL, Albiger B, et al. Worsening epidemiological situation of carbapenemase-producing Enterobacteriaceae in Europe, assessment by national experts from 37 countries, July 2018. Euro Surveill 2019;24(9).

5. Nordmann P, Naas T, Poirel L. Global spread of Carbapenemase-producing Enterobacteriaceae. Emerg Infect Dis 2011;17(10):1791-8.

6. Nordmann P, Poirel L. The difficult-to-control spread of carbapenemase producers among Enterobacteriaceae worldwide. Clin Microbiol Infect 2014:20(9):821-30.

7. Iacchini S, Sabbatucci M, Gagliotti C, Rossolini GM, Moro ML, Iannazzo S, et al. Bloodstream infections due to carbapenemase-producing Enterobacteriaceae in Italy: results from nationwide surveillance, 2014 to 2017. Euro Surveill 2019;24(5).

8. World health OrganizationGuidelines for the prevention and control of carbapenem-resistant enterobacteriaceae, acinetobacter baumannii and pseudomonas aeruginosa in health care facilities. Geneva: World Health Organization; 2017. WHO Guidelines Approved by the Guidelines Review Committee p. 74.

9. Magiorakos AP, Burns K, Rodriguez Bano J, Borg M, Daikos G, Dumpis U, et al. Infection prevention and control measures and tools for the prevention of entry of carbapenem-resistant Enterobacteriaceae into healthcare settings: guidance from the European Centre for Disease Prevention and Control. Antimicrob Resist Infect Control 2017;6:113.

10. Centers for Disease Control and Prevention: National Center for Emerging and Zoonotic Infectious Diseases, Division of Healthcare Quality. Facility Guidance for Control of Carbapenem Resistant Enterobacteriaceae (CRE) November 2015 Update; 2015. [cited 2019 May 27]. Available from: https://www.cdc.gov/hai/ pdfs/cre/CRE-guidance-508.pdf.

11. Tacconelli E, Carrara E, Savoldi A, Harbarth S, Mendelson M, Monnet DL, et al. Discovery, research, and development of new antibiotics: the WHO priority list of antibiotic-resistant bacteria and tuberculosis. Lancet Infect Diseas 2018;18(3):318-27

12. Dickstein Y, Edelman R, Dror T, Hussein K, Bar-Lavie Y, Paul M. Carbapenem-resistant Enterobacteriaceae colonization and infection in critically ill patients: a retrospective matched cohort comparison with non-carriers. J Hosp Infect 2016;94(1):54-9.

13. Giannini MA, Gilliam C, Owings A, Glover B, Gipson M, Hakim H. Does colonization with carbapenem-resistant Enterobacteriaceae correlate to infection? Am J Infect Control 2017;45(6):S37.

14. Tischendorf J, de Avila RA, Safdar N. Risk of infection following colonization with carbapenem-resistant Enterobactericeae: a systematic review. Am J Infect Control 2016:44(5):539-43.

15. McConville TH, Sullivan SB, Gomez-Simmonds A, Whittier S, Uhlemann AC. Carbapenem-resistant Enterobacteriaceae colonization (CRE) and subsequent risk of infection and 90-day mortality in critically ill patients, an observational study. PLoS One 2017;12(10):e0186195.

16. Falagas ME, Tansarli GS, Karageorgopoulos DE, Vardakas KZ. Deaths attributable to carbapenem-resistant Enterobacteriaceae infections. Emerg Infect Dis 2014;20(7):1170-5. 
17. Stoesser N, Xayaheuang S, Vongsouvath M, Phommasone K, Elliott I, Del Ojo Elias C, et al. Colonization with enterobacteriaceae producing ESBLs in children attending pre-school childcare facilities in the Lao People's Democratic Republic. J Antimicrob Chemother 2015;70(6):1893-7.

18. Suwantarat N, Carroll KC. Epidemiology and molecular characterization of multidrug-resistant Gram-negative bacteria in Southeast Asia. Antimicrob Resist Infect Control 2016;5:15.

19. Turner P, Pol S, Soeng S, Sar P, Neou L, Chea P, et al. High prevalence of antimicrobial-resistant gram-negative colonization in hospitalized cambodian infants. Pediat Infect Diseas J 2016;35(8):856-61.

20. Le NK, Hf W, Vu PD, Khu DT, Le HT, Hoang BT, et al. High prevalence of hospital-acquired infections caused by gram-negative carbapenem resistant strains in Vietnamese pediatric ICUs: a multi-centre point prevalence survey. Medicine (Baltimore) 2016;95(27):e4099.

21. Phu VD, Wertheim HF, Larsson M, Nadjm B, Dinh QD, Nilsson LE, et al. Burden of hospital acquired infections and antimicrobial use in vietnamese adult intensive care units. PLoS One 2016;11(1):e0147544.

22. Berglund B, Hoang NTB, Tarnberg M, Le NK, Welander J, Nilsson M, et al. Colistin- and carbapenem-resistant Klebsiella pneumoniae carrying MCR-1 and blaOXA-48 isolated at a paediatric hospital in Vietnam. $J$ Antimicrob Chemother 2018;73(4):1100-2.

23. Berglund B, Hoang NTB, Tarnberg M, Le NK, Svartstrom O, Khu DTK, et al. Insertion sequence transpositions and point mutations in mgrB causing colistin resistance in a clinical strain of carbapenem-resistant Klebsiella pneumoniae from Vietnam. Int J Antimicrob Agents 2018;51(5):789-93.

24. Zarb P, Coignard B, Griskeviciene J, Muller A, Vankerckhoven V, Weist K, et al. The European Centre for Disease Prevention and Control (ECDC) pilot point prevalence survey of healthcare-associated infections and antimicrobial use. Euro Surveill 2012;17(46).

25. Richter SS, Marchaim D. Screening for carbapenem-resistant Enterobacteriaceae: who, when, and how? Virulence 2017;8(4):417-26.

26. Viau R, Frank KM, Jacobs MR, Wilson B, Kaye K, Donskey CJ, et al. Intestinal carriage of carbapenemase-producing organisms: current status of surveillance methods. Clin Microbiol Rev 2016;29(1):1-27.

27. Chiotos K, Tamma PD, Flett KB, Karandikar MV, Nemati K, Bilker WB, et al. Increased 30-day mortality associated with carbapenem-resistant enterobacteriaceae in children. Open Forum Infect Dis 2018;5(10) ofy222.
28. Nour I, Eldegla HE, Nasef N, Shouman B, Abdel-Hady H, Shabaan AE. Risk factors and clinical outcomes for carbapenem-resistant Gram-negative late-onset sepsis in a neonatal intensive care unit. J Hosp Infect 2017;97(1):52-8.

29. Peters L, Olson L, Khu DTK, Linnros S, Le NK, Hanberger H, et al. Multiple antibiotic resistance as a risk factor for mortality and prolonged hospital stay: a cohort study among neonatal intensive care patients with hospital-acquired infections caused by gram-negative bacteria in Vietnam. PLoS One 2019;14(5):e0215666.

30. Logan LK, Weinstein RA. The Epidemiology of Carbapenem-Resistant Enterobacteriaceae: the Impact and Evolution of a Global Menace. J Infect Dis 2017;215(suppl_1):S28-36.

31. Zhao ZC, Xu XH, Liu MB, Wu J, Lin J, Li B. Fecal carriage of carbapenem-resistant Enterobacteriaceae in a Chinese university hospital. Am J Infect Control 2014;42(5):e61-4.

32. Stewardson AJ, Marimuthu K, Sengupta S, Allignol A, El-Bouseary M, Carvalho MJ, et al. Effect of carbapenem resistance on outcomes of bloodstream infection caused by Enterobacteriaceae in low-income and middle-income countries (PANORAMA): a multinational prospective cohort study. Lancet Infect Diseas 2019.

33. Haverkate MR, Platteel TN, Fluit AC, Cohen Stuart JW, Leverstein-van Hall MA, Thijsen SFT, et al. Quantifying within-household transmission of extended-spectrum beta-lactamase-producing bacteria. Clin Microbiol Infect 2017;23(1):46 e1-e7.

34. Centers for Disease Control and Prevention Guidance for control of infections with carbapenem-resistant or carbapenemase-producing Enterobacteriaceae in acute care facilities. MMWR Morb Mortal Wkly Rep 2009;58(10):256-60.

35. Ben-David D, Masarwa S, Adler A, Mishali H, Carmeli Y, Schwaber MJ. A national intervention to prevent the spread of carbapenem-resistant Enterobacteriaceae in Israeli post-acute care hospitals. Infect Control Hosp Epidemiol 2014;35(7):802-9.

36. Hussein K, Rabino G, Eluk O, Warman S, Reisner S, Geffen Y, et al. The association between infection control interventions and carbapenem-resistant Enterobacteriaceae incidence in an endemic hospital. J Hosp Infect 2017;97(3):218-25. 